\title{
Investigation of the Ablation Rate of Stainless Steel by means of few Ultrashort Laser Pulses up to $150 \mathrm{~J} / \mathrm{cm}^{2}$
}

\author{
Dennis Haasler, Tobias Schneider and Reinhart Poprawe \\ Chair for Laser Technology LLT, RWTH Aachen University, Germany \\ E-mail: dennis.haasler@llt.rwth-aachen.de
}

\begin{abstract}
The availability of high power ultrafast lasers for material processing enables new applications such as deep hole drilling. To reach high ablation rates and high aspect ratios, fluences in the order of magnitude of $100 \mathrm{~J} / \mathrm{cm}^{2}$ are required. As this range has rarely been described in literature, a detailed study of the ablation rate for stainless steel is presented here. At a mean fluence of $\sim 1.3 \mathrm{~J} / \mathrm{cm}^{2}$ a molten phase is observed in the center of the hole which then extends over the entire ablated area for increasing fluences. Due to the onset of a melt layer a melt supported ablation superimposes the anticipated second ablation regime and hence no logarithmic dependence of the ablation depth is visible for higher fluences. For fluences of $10 \mathrm{~J} / \mathrm{cm}^{2}$ and higher the entire area is subject to a melt supported ablation. In addition, in this fluence range only the deposition of a few pulses at a higher repetition rate can boost the ablation rate up to a factor of 10 due to heat accumulation.
\end{abstract}

DOI: $10.2961 /$ jlmn.2019.03.0007

Keywords: fluence, ablation regimes, ablation depth, usp-processing, heat accumulation, melt

\section{Introduction}

The demonstration of the first self-mode-locked Ti-sapphire laser in 1990 lead to much more practical and versatile ultrashort-pulse (usp) sources in the femtosecond (fs)-regime compared to the previously used dye-lasers [1]. This development triggered diverse investigations of new applications in the following years with fs-lasers. The first publications for material processing with such lasers have compared the ablation rate and the quality in various materials to the results of nanosecond laser pulses [2-7]. In detail, different types of materials like metals, semiconductors, dielectrics, ceramics and biomaterials [2,3], damage and ablation thresholds [3-5, 7], influence of ambient pressure [6] and different wavelengths from near infrared to uv-radiation [3, $4,7]$ have been investigated. In 1996, Momma and Chichkov $[8,9]$ did an extensive study for the ablation rate per pulse from femtosecond to nanosecond pulses on steel and from low to very high fluences up to $10^{4} \mathrm{~J} / \mathrm{cm}^{2}$. For high fluences with femtosecond pulses, they suggest that a liquid phase is the created during each pulse which leads to drops on the surface and a distinct recast layer in the produced groove. They concluded that no considerable advantages in material processing compared to longer pulses exist for high fluences. For low fluences, a precise ablation with only some - previously vaporized - material deposition on the surface was observed. In a subsequent paper, they focused on the low fluence ablation on different metals [9]. One year later, the group published their next paper including a graph with the ablation depth per pulse as a function of the fluence, revealing two ablation regimes. They also introduced a simple theoretical model to describe those experimental results which is now commonly known as the two-temperature model. In a first step, the photons of a laser pulse transfer their energy to the electrons (via inverse Bremsstrahlung) which in turn thermalize the phonon lattice afterwards. On short timescales - below the electron phonon coupling time $(<\sim 10 \mathrm{ps})$
- the energy or 'temperature' of the electrons differs from the temperature of the lattice.

Therefore, they introduced the two temperature model in which they describe the temperature of the electrons and the lattice separately. Based on this model, a logarithmic dependence for the ablation depth per pulse is derived and has the form:

$$
\begin{array}{ll}
L \simeq \delta \ln \left(F / F_{t h}^{\delta}\right) & (\delta \gg l) \\
L \simeq l \ln \left(F / F_{t h}^{l}\right) & (\delta \ll l)
\end{array}
$$

With: $\delta=$ optical penetration depth, $l=$ electron heat conduction depth, $F_{t h}=$ corresponding threshold fluence

Depending on the fluence, the optical or electron penetration depth is predominant and determines the ablation per pulse, resulting in the two observed regimes. [10-11]

Le Harzic et al. [12] showed the dependency of the gradient and threshold fluences for both regimes on different metals and pulse durations between $100 \mathrm{fs}$ and $4.5 \mathrm{ps}$. For steel and aluminum, an increase in the gradient for shorter pulses is observed. It is also shown, that the fluence threshold increases with increasing pulse duration mainly for the low fluence regime.

Those results have been confirmed in numerous publications where the threshold fluence and penetration depth for various pulse durations and materials have been determined [7, 12-23] This model is to date accepted to describe the ablation depth per pulse typically up to $10 \mathrm{~J} / \mathrm{cm}^{2}$.

However, methods of gaining the ablation rate per pulse, pulse durations and other parameters are often differing, leading to a significant variation of resulting threshold values and penetration depths. For example, the ablation threshold was calculated in diverse publications by measuring: 
- $\quad$ ablation depth for a selected number of pulses ○ on one spot $[9-10,14-15,17,20]$,

- along a groove $[12,14,15,18]$,

o over an area [29],

- the ablated diameter [13-14, 22-23].,

- the amount of numbers to drill through a sample $[8,9,16]$.

Since commercially available ultrashort pulsed (usp) laser sources have reached high average powers of several $100 \mathrm{~W}$ in the last years, new applications for usp processing have become of interest. [24, 25] In case of deep hole drilling, a usp based process has the potential of producing a nearly melt and defect free hole with a high geometrical accuracy [26-28]. To manufacture those holes a high pulse energy in the order of magnitude of millijoule is crucial to enable:

- Ablation at steep hole wall

- Ablation out of focus

- High ablation rate

- Low residual heat

- Low repetition rate

Potentially feasible spot sizes for deep hole drilling result in fluences in the range of ten to hundreds of $\mathrm{J} / \mathrm{cm}^{2}$. Unfortunately, only very few works investigate this parameter range:

Mingareev [17] has investigated the ablation depth per pulse up to $300 \mathrm{~J} / \mathrm{cm}^{2}$ with few or single shots but has rarely described the results. Nedialkov et al. [20] show the ablation depth per pulse for fluences up to $100 \mathrm{~J} / \mathrm{cm}^{2}$ and compare them with molecular dynamic simulations. A fit and description was only done until a fluence of $10 \mathrm{~J} / \mathrm{cm}^{2}$. Also, both Migareev and Nedialkov present very few data points in the region of interest. Only Dausinger [15] gives a more detailed graph covering the range from $0.4-200 \mathrm{~J} / \mathrm{cm}^{2}$. However, he does not discuss the course of the graph. Other of his findings as the significant change of the hole diameter by a factor of 10 for an increasing fluence indicate the existence of additional (drilling based) effects that probably also modify the ablation rate.

It is also evident that at a certain fluence a liquid phase occurs which remains after the process as a melt layer. For the ablation at extremely high fluences up to $10^{4} \mathrm{~J} / \mathrm{cm}^{2}$, the findings from Momma and Chichkov [8,9] give an insight with the afore mentioned results of producing a layer of melt with each pulse. Nedialkov et al. [20] already observes at $10 \mathrm{~J} / \mathrm{cm}^{2}$ a distinct melt layer in and around the hole. For an even lower fluence of $4.3 \mathrm{~J} / \mathrm{cm}^{2}$ and only a few pulses, a molten region in the center of ablated depression is described by Mannion et al. [22].

According to molecular dynamics simulations, a liquid phase is nearly always present during ultrafast laser processing. Even below the threshold fluence, the laser radiation leads to melting of a few $10 \mathrm{~nm}$ thick layer of material. For high fluences, material ablation is dominated by phase explosion of the overheated liquid phase with melting depths in the range of $100 \mathrm{~nm}[20,30]$. Those ablation mechanisms are already present at typical fluences for laser material processing while no considerable melt layer remains after the process. The word melt layer will be used in this paper when a smooth and ripple free melt layer is observed after the process and no typical micro-structured surface is present in this region anymore.

Therefore, one aspect of this publication is to investigate the course of the ablation rate up to fluences of $150 \mathrm{~J} / \mathrm{cm}^{2}$ in detail for stainless steel. Additionally, the threshold for the creation of a smooth and ripple free melt layer and the consequences on the course of the second regime shall be examined. To minimize other influences, all experiments are conducted by placing only a few pulses with a low repetition rate on one spot. Other potentially influencing parameters like the spot size or the number of pulses are also monitored at those experiments. The experiments are analyzed by measuring the ablation depth and volume. Those investigations shall give an insight which parameters actually modify the ablation rate.

In a second step, the occurrence of a melt layer at lower fluences due to heat accumulation is investigated. Both the number of pulses and the repetition rate are examined in this step. High power lasers for material processing often have a higher pulse duration than the laser beam sources used for the fundamental research in the first part. Therefore and first of all, the results that are obtained before with a pulse duration of $100 \mathrm{fs}$ are validated for a higher pulse duration of $2 \mathrm{ps}$ Also, the influence of process gas on the ablation rate is determined as high power applications otherwise could harm the optics without any process gas.

\section{Experimental Procedure}

Two usp laser beam sources are utilized for the experiments. For the fundamental experiments, a laser beam source from Coherent (type Libra) is employed. In this case a maximum pulse energy of $E_{P}=1.5 \mathrm{~mJ}$ is focused by a lens with $\mathrm{f}=175 \mathrm{~mm}$ on the samples, resulting in a focal radius of $w_{0}=18 \mu \mathrm{m}$. A second spot size radius of $w_{0}=26 \mu \mathrm{m}$ is achieved by a telescope in the raw beam. Due to the small repetition rate no process gas is utilized in this set of experiments.

The AMPHOS 400 flex is used for the other experiments, as it has a higher average power and therefore is more suitable for material processing. The maximum pulse energy of $E_{P}=0.85 \mathrm{~mJ}$ can be achieved for repetition rates of $\mathrm{f}$ $\leq 400 \mathrm{kHz}$. The beam is focused by an f-theta optic (JENar, $\mathrm{f}=160 \mathrm{~mm}$ ) onto the samples which are mounted on a xystage. Three different spot sizes are tested, $w_{0}=28 \mu \mathrm{m}, w_{0}=$ $16 \mu \mathrm{m}$ by increasing the raw beam with a telescope and $w_{0}=$ $50 \mu \mathrm{m}$ by defocusing the spot. A coaxial process gas nozzle

Table 1 Utilized parameters of the laser beam sources

\begin{tabular}{ccc}
\hline Specifications & $\begin{array}{c}\text { Amphos 400 } \\
\text { flex }\end{array}$ & Libra \\
\hline Wavelength & $1030 \mathrm{~nm}$ & $800 \mathrm{~nm}$ \\
Max. Average Power & $340 \mathrm{~W}$ & $1.5 \mathrm{~W}$ \\
Pulse Duration & $2 \mathrm{ps}$ & $100 \mathrm{fs}$ \\
Repetition Rate & $1 \mathrm{~Hz}-400 \mathrm{kHz}$ & $5 \mathrm{~Hz}$ \\
Max. Pulse Energy & $0.85 \mathrm{~mJ}$ & $1.5 \mathrm{~mJ}$ \\
Beam Quality $\left(M^{2}\right)$ & 1.6 & 1.2 \\
\hline
\end{tabular}




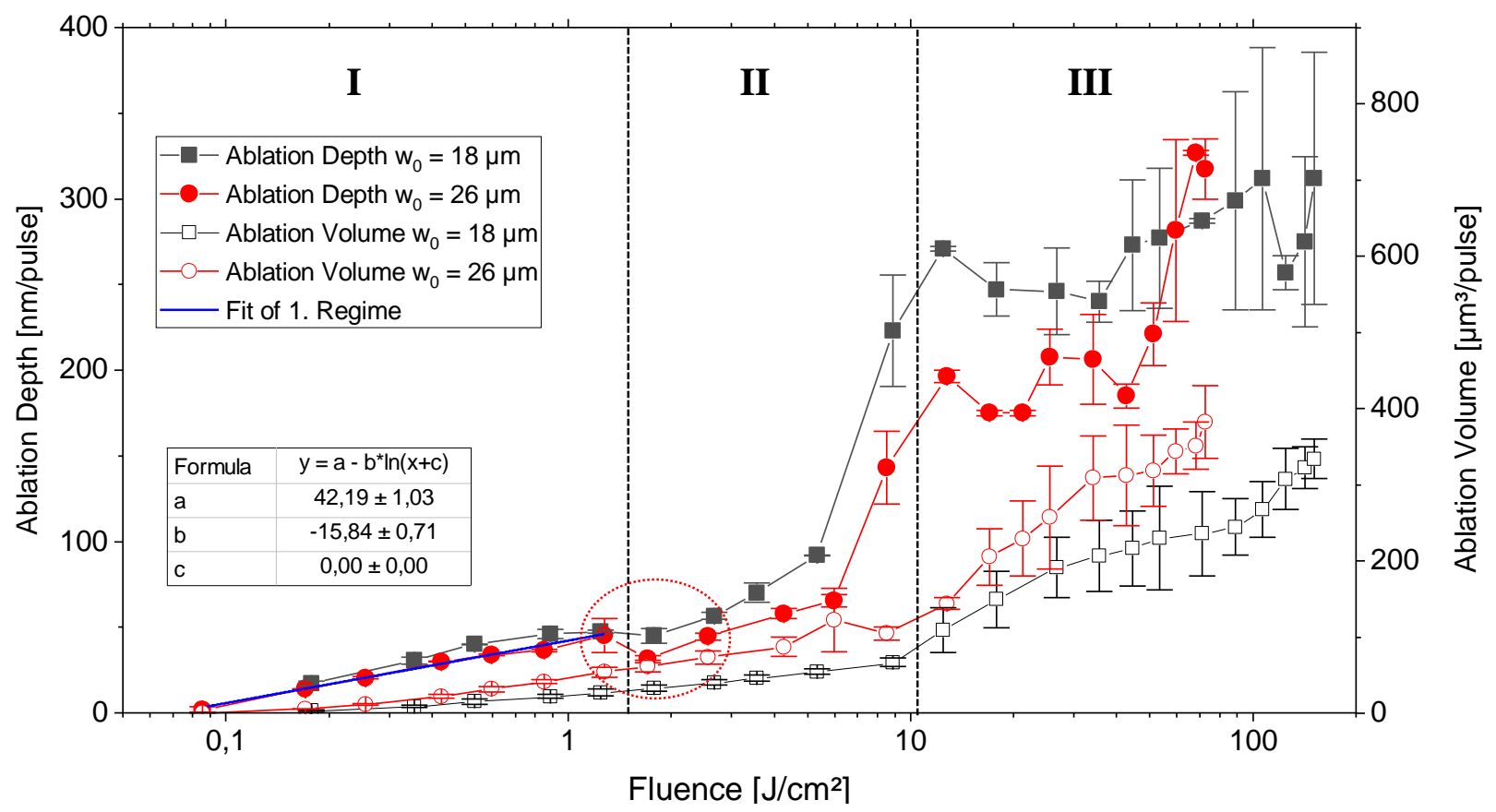

Fig. 1 Ablation depth and ablation volume per pulse as a function of the fluence for stainless steel. The dotted circle highlights a common dip in the course for the maximum ablation depth (Laser: Libra, $\lambda=800 \mathrm{~nm}, \mathrm{f}=5 \mathrm{~Hz}, \mathrm{t}_{\mathrm{p}}=100 \mathrm{fs}, \mathrm{w}_{0}=18 \mu \mathrm{m}: 25 \& 50$ pulses, $\mathrm{w}_{0}=26 \mu \mathrm{m}$ : $50 \& 100$ pulses).
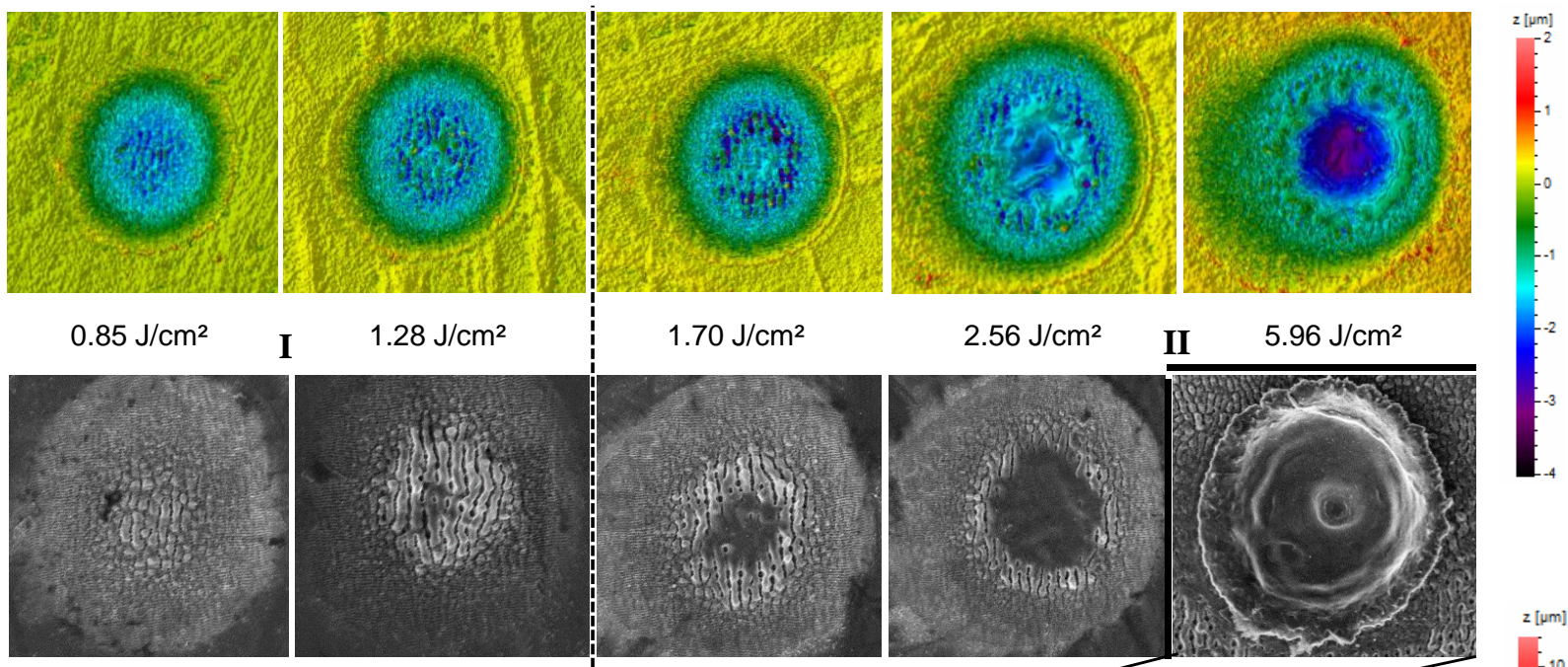

$$
\text { I } \quad 1.28 \mathrm{~J} / \mathrm{cm}^{2}
$$

$1.70 \mathrm{~J} / \mathrm{cm}^{2}$

$2.56 \mathrm{~J} / \mathrm{cm}^{2}$

II $\quad 5.96 \mathrm{~J} / \mathrm{cm}^{2}$
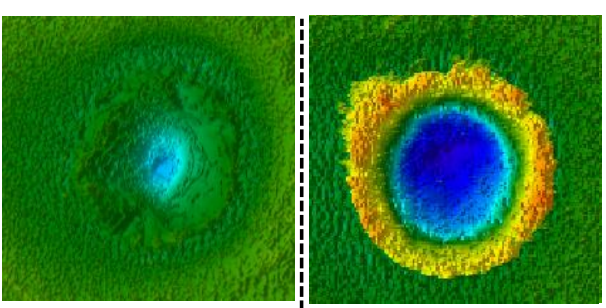

$12.78 \mathrm{~J} / \mathrm{cm}^{2}$

$8.52 \mathrm{~J} / \mathrm{cm}^{2}$

$50 \mu \mathrm{m}$
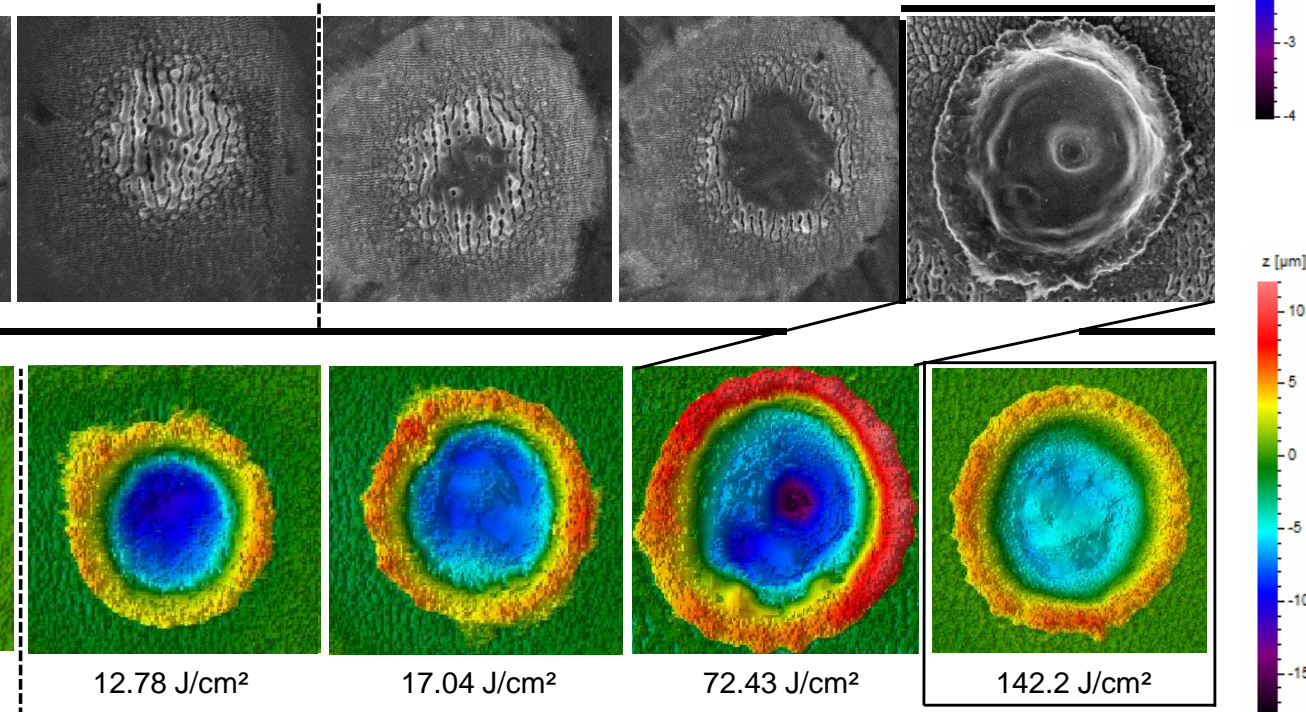

$17.04 \mathrm{~J} / \mathrm{cm}^{2}$

III

Fig. 2 White light interferometer (WLI) images of the produced holes in stainless steel. The depth is displayed in pseudo-colors and its scale is differing in the images. All shown holes have been created with a spot of $\mathrm{w}_{0}=26 \mu \mathrm{m}$ except the last one $\left(\mathrm{w}_{0}=18 \mu \mathrm{m}\right)(\mathrm{Laser}$ : Libra, $\lambda=800 \mathrm{~nm}, \mathrm{t}_{\mathrm{p}}=100 \mathrm{fs}, \mathrm{f}=5 \mathrm{~Hz}, 50$ pulses [last one: 25$]$ ). 
is placed above the sample to provide pressured air at 5 bar in this setup. The spot sizes are measured with a camera $\left(1 / \mathrm{e}^{2}\right)$. All employed parameters of the two laser beam sources are also listed in Table 1.

All experiments are conducted with samples of stainless steel (1.4301) which are polished until a roughness of below $\mathrm{Ra}=30 \mathrm{~nm}$ is achieved. The ablation rate is investigated by focusing a few laser pulses on the sample surface while maintaining the following constraints

- The aspect ratio of the produced hole/depression should be ideally well below 0.5 to exclude any drill based phenomena. [23]

- A minimum amount of pulses is essential to obtain a reproducible result, as the ablation especially for higher fluence fluctuates extensively during the first pulses. Typically, good results can be obtained for 20 pulses and more. [22]

The analysis of the holes to determine the ablation depth and the ablation volume has been performed with a white light interferometer (WLI) (NewView 7300 from Zygo) and a laser scanning microscope (LSM) (VK-9700 from Keyence). Additionally, images with a scanning electron microscope (SEM) are made for selected holes.

\section{Experimental Results}

\subsection{Ablation Regimes at $\mathbf{1 0 0}$ fs}

In Figure 1 the ablation depth and ablation volume per pulse for two different spot diameters are shown for varying fluences at a pulse duration of $100 \mathrm{fs}$. The printed fluence ranges from the ablation threshold of stainless steel and rising up to about $150 \mathrm{~J} / \mathrm{cm}^{2}$. The data points are acquired by experiments that are conducted with two different numbers of pulses for each spot diameter to also include a potential influence of this parameter. For lower fluences up to about $1 \mathrm{~J} / \mathrm{cm}^{2}$ (region I) the first regime is present, leading to a logarithmic rise of the maximum ablation depth. A fit of those data points is also shown in the graph and yields to the values displayed in Table 2. For fluences greater than $\sim 1 \mathrm{~J} / \mathrm{cm}^{2}$, the second regime begins according to literature $[12,16,17,19$, $21,29]$. Interestingly, this second region starts with a small dip in the ablation depth (see dotted circle in Figure 1). Also, the slope of the ablation depth for fluences up to $6 \mathrm{~J} / \mathrm{cm}^{2}$ is only slightly higher with respect to the first regime. Subsequently, between 6 and $12 \mathrm{~J} / \mathrm{cm}^{2}$, the ablation depth rises by a factor of three. For a higher fluence the ablation depth further rises on average but is subject to a high variance which has not been present before. Both spot diameters show a very similar course, but the ablation depth for the smaller spot tends to be slightly higher after the first regime.

However, if the ablation volume is considered, a monotonic and roughly linear course is observed for high fluences above $12 \mathrm{~J} / \mathrm{cm}^{2}$. The ablation volume for the bigger spot is significantly higher compared to the smaller spot because a greater area is irradiated. Assuming that the ablation volume also roughly scales with the irradiated area, the ablated volume should be approximately twice as high for the bigger spot.

With the aid of WLI and SEM images as seen in Figure 2 the course of the graph is better understandable. In region I which displays the first regime, the ablation rate is based on the optical penetration depth of the photons and corresponds well with the parameters in other publications (see
Table 2). The images of the holes in this region present a typical micro-structured surface, also showing the formation of ripples for higher fluences (i.e. $\mathrm{F}=1.26 \mathrm{~J} / \mathrm{cm}^{2}$ ). The third picture shows the first hole which is counted to region II, which has a lower depth compared to the holes before. This is not a statistical outlier, as one might think solely based on the graph. In all previous holes with lower fluences, the highest depth was measured in the 'valleys' of the ripples. In the currently mentioned picture the center of the hole where the highest local fluence is applied - shows a much more uniform and smooth surface without ripples anymore. Hence, this dip marks the point where the peak fluence is high enough to clearly produce melt for the first time. The local maximum depth in the corrugated surface is then replaced by the depth of a smooth surface. The total volume ablated at this fluence is still higher than for the smaller fluences which confirms that this is not just a coincidence. Having a closer look on the SEM images in Figure 2, the creation of a melt layer during ablation actually begins at about $1.3 \mathrm{~J} / \mathrm{cm}^{2}$. Especially the darker color of the surface in the region which represents the melt layer for higher fluences, suggest that this fluence is probably the true starting point. For an increasing fluence, the fraction of the hole in which melt is produced increases $\left(2.56-12.78 \mathrm{~J} / \mathrm{cm}^{2}\right)$. Region II is therefore defined here as the fluence range in which both the vaporization based ablation and the melt supported ablation are coexistent.

In Region III, at about $10 \mathrm{~J} / \mathrm{cm}^{2}$ and above, melt is produced over the entire hole area and therefore dominates the ablation process. Regarding the WLI images, the ablated surface is rather smooth compared to region I and is also accompanied by a molten ring around the hole. Up to $142 \mathrm{~J} / \mathrm{cm}^{2}$, the principal shape of the hole does not change. In this region, the ablation rate is influenced to some extent by the molten material and thus explains the variations in the ablation depth for high fluences in Figure 1.

\subsection{Discussion of the observations}

At first sight, this graph looks different for fluences of $1 \mathrm{~J} / \mathrm{cm}^{2}$ and above compared with graphs from previous publications which examined the two regimes. A close look at the graphs published in $[16,19,21]$ reveals a very similar course of the graph than shown here. Unfortunately, this region is not further discussed.

For low fluences (region I) the logarithmic dependence of the ablation depth is confirmed by the results. The threshold value and penetration depth is in the same range as in other publications (see Table 2). Roughly at the beginning of the second regime, the course of the graph looks different compared to the expected appearance with the two logarithmic regimes. Up to a fluence of $\sim 6 \mathrm{~J} / \mathrm{cm}^{2}$, the inclination does not significantly change and also a dip is present at the transition from the first regime. With the aid of WLI and SEM images the reason for this dip and the unchanged inclination can be deduced. Precisely the data point that shows the $\operatorname{dip}\left(\mathrm{F}=1.7 \mathrm{~J} / \mathrm{cm}^{2}\right)$ displays a molten phase in the center for the first time. The dip seems to be marginal and is below the error bars. But it has been observed in each set of experiments and therefore indicates the presence of melt without the aid of SEM-images. Assuming a Gaussian beam shape of the focal spot, a peak fluence of $2.6-3.4 \mathrm{~J} / \mathrm{cm}^{2}$ leads to 
the creation of a melt layer. For an increasing fluence, the region in which a melt layer exists increases likewise.

With the steep rise of the ablation rate towards region III (by a factor of 3), the melt supported ablation starts to dominate.

This rise is probably caused by the fact that the holes produced by lower fluences have not reached their maximum depth before. A lower ablation depth is measured if the area which is ablated in the melt supported regime is too small, the surface tension of the molten material prevents it from leaving the center of the hole. Also, the created vaporization pressure might not be high enough to accelerate the liquid yet. Here again the ablation volume suggests this explanation, as the inclination of this graph in this region does not alter much compared to the previous course.

As a result, especially region II strongly depends on the spatial beam shape as this defines the composition of the vaporization dominated and melt supported ablation process. Hence, the course of the ablation as a function of the fluence might differ in region II while in region I and III an identical course is displayed. It should also be kept in mind that in case of a top-hat beam profile region II should not exists. Also, due to the much steeper hole walls, the melt transport that might be present for higher fluences could behave differently and ultimately result in a different ablation curve. In region III the entire hole area is ablated with a melt supported ablation process, therefore leading to higher variations as seen in the increasing difference between single points and elongated error bars. As the ring of molten material around the hole indicates, some acceleration of molten material is present after each pulse similar to the drilling process principle with long pulsed lasers. As the result, the introduction of melt dynamics greatly boosts the variance of the process. The plateau seen in region III for high fluences is probably caused by the scattering of the laser pulses in a cold atmospheric plasma due to the high intensities. Hence, the pulse energy is redistributed to a bigger area, reducing the ablation depth but not affecting the ablated volume. As described before, the ablation volume is not subject to those strong variations. Even though the variance of the data points increase in region III as well, the general trend is much smoother and roughly linear in the graph. Therefore, it might be more suitable for describing the ablation rate at high fluences with the aid of the ablation volume for future investigations. The ablation depth is obviously of no use for higher fluences as the second regime cannot be used to describe the ablation rate in case of stainless steel anymore.

With the occurrence of a melt layer already at such small fluences of about $1.3 \mathrm{~J} / \mathrm{cm}^{2}$, the second regime is superimposed by the melt supported ablation already from the beginning onwards. This is the reason why the second regime cannot be observed in this study on stainless steel. Also, Nedialkov et al. [20] came to similar results based on experiments and molecular dynamics simulations. As stated before, he also observed a distinct molten phase at $10 \mathrm{~J} / \mathrm{cm}^{2}$. He explained his findings with two mechanisms. According to his simulations, at higher fluences $\left(>0.5 \mathrm{~J} / \mathrm{cm}^{2}\right)$ the material overheats which results in a steep rise of the pressure in the material. This force then accelerates the melt out of the hole. But in combination with a larger affected volume due to the increased electron penetration depth, the energy is transported further into the material. As a result, this leads to a higher molten volume and less overheated material which eventually shifts the threshold for the recoil pressure to expel the melt to higher fluences. This fits to the previous observations which first show the creation of a molten layer and only later the acceleration of the melt with a steep increase in the ablation depth (see Figure 1 and 2). With an increase of the molten volume, a bigger fraction of the molten material remains in the hole after the ablation process which leads eventually to a visible melt layer.

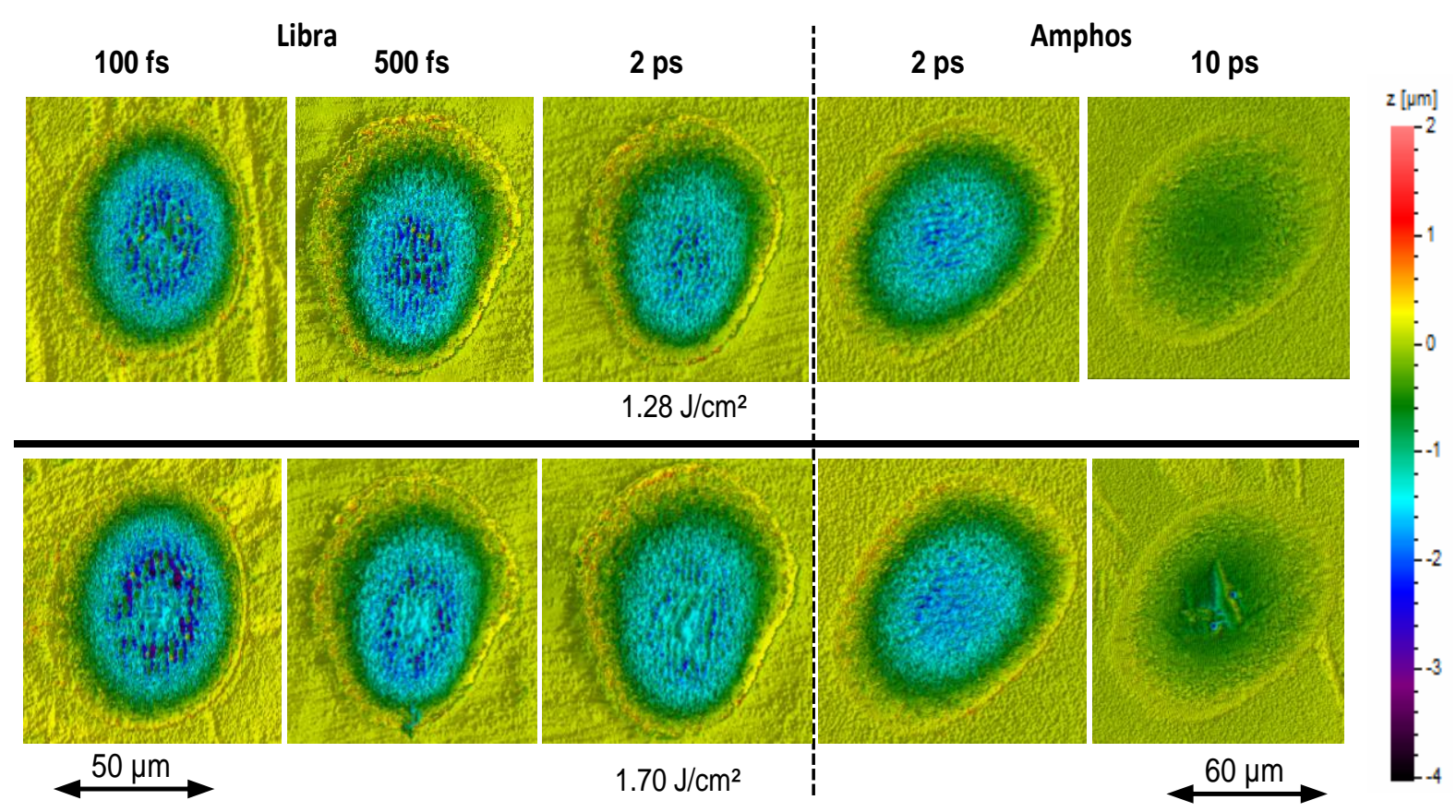

Fig. 3 WLI images of holes created with a fluence just below the threshold $\left(1.28 \mathrm{~J} / \mathrm{cm}^{2}\right)$ and over the threshold $\left(1.70 \mathrm{~J} / \mathrm{cm}^{2}\right)$ after which a melt layer is generated. The depth is displayed in pseudo-colors and its scale is differing in the images. In all cases 50 pulses are used (Libra: $\lambda=800 \mathrm{~nm}, \mathrm{t}_{\mathrm{p}}=100 \mathrm{fs}, \mathrm{f}=5 \mathrm{~Hz}, \mathrm{w}_{0}=26 \mu \mathrm{m}$. Amphos: $\left.\lambda=1030 \mathrm{~nm}, \mathrm{t}_{\mathrm{p}}=2 \mathrm{ps}, \mathrm{f}=1000 \mathrm{~Hz}, \mathrm{w}_{0}=28 \mu \mathrm{m}\right)$. 


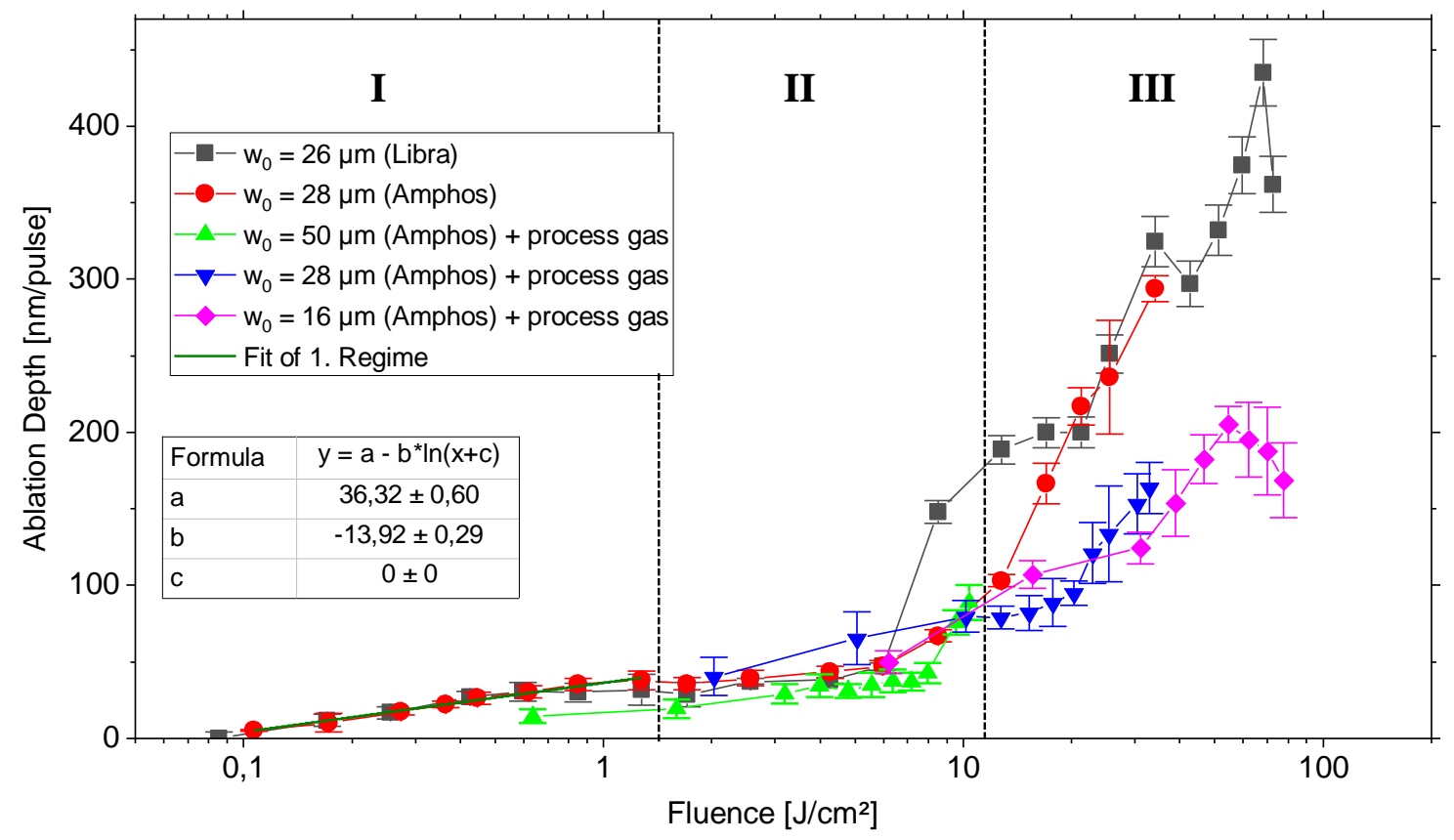

Fig. 4 Ablation depth and ablation volume per pulse as a function of the fluence for stainless steel (Libra: $\lambda=800 \mathrm{~nm}, \mathrm{t}_{\mathrm{p}}=100 \mathrm{fs}, \mathrm{f}=5 \mathrm{~Hz}$, $\mathrm{w}_{0}=26 \mu \mathrm{m}$. Amphos: $\lambda=1030 \mathrm{~nm}, \mathrm{t}_{\mathrm{p}}=2 \mathrm{ps}, \mathrm{f}=1000 \mathrm{~Hz}, 50$ pulses $)$.

Zhigilei [30] came to the same assumption when comparing his molecular dynamics simulations with experimental results. The simulations are made for nickel but it is assumed to react similar due to similar physical properties. Their calculated melting depths for higher fluences are in the same order than the ablation depth and also the fluence dependence on the melting depth is consistent with the experiments. They could not directly compare the ablation depth of the simulations with the experimental results, as the simulations did not include the pressure gradients from the recoil pressure which accelerates the melt out of the hole.

The threshold fluence for the creation of a melt layer was observed in all experiments connected to Figure 1 and is also confirmed for pulse durations of $t_{p}=500 \mathrm{fs}$ and $2 \mathrm{ps}$. This is illustrated in Figure 3 with additional WLI images that show the surface of the ablated areas directly at the threshold $\left(1.28 \mathrm{~J} / \mathrm{cm}^{2}\right)$ and after $\left(1.70 \mathrm{~J} / \mathrm{cm}^{2}\right)$ the onset of a melt layer. In case of $10 \mathrm{ps,} \mathrm{the} \mathrm{morphology} \mathrm{of} \mathrm{the} \mathrm{radiated}$ surface changes. The observed ripples are have a significantly smaller wavelength compared to all other examined pulse durations. At the threshold fluence still a molten surface evolves but this one also differs with respect to the other pulse durations. Thus, it is unclear whether the aforementioned findings can also be applied at a pulse duration of $10 \mathrm{ps}$.

In addition, the depression created with 2 ps pulses is also done on the second laser system for comparison. This is the only example in which a melt layer is not clearly visible on the images. A potential explanation could be the different wavelength of the second setup (1030 nm instead of $800 \mathrm{~nm}$ ). The same experiments were conducted on a copper sample to cross-check those findings for stainless steel. The discussed, clearly melt based phenomena have not been observed up to fluences of about $50 \mathrm{~J} / \mathrm{cm}^{2}$.

\subsection{Ablation regimes at $2 \mathrm{ps} \&$ influence process gas}

The same investigations conducted for $100 \mathrm{fs}$ pulses are performed for a pulse duration of 2 ps and presented in Figure 4. As shown before, the development of a melt layer takes place still at the same fluence. However, the use of the Amphos laser source results in a different surface structure despite the same pulse duration. Therefore, both laser beam sources are compared in the graph. To provide a reference for the second step in which the impact of a higher average power is analyzed, some of the experiments are conducted with the aid of process gas.

The reference experiments are performed with the same setup as for the experiments in Figure 1 but with a pulse duration of 2 ps. The course of this graph is very close to the course of the $100 \mathrm{fs}$ pulses. For low fluences in region I, a linear fit is calculated again and the threshold fluence and

Table 2 Threshold fluence and optical penetration depth for the first regime of both pulse durations and in comparison with data from literature for stainless steel

\begin{tabular}{ccc}
\hline $\begin{array}{c}\text { Source/ } \\
\text { Pulse duration }\end{array}$ & $\begin{array}{c}\text { Threshold } \\
\text { Fluence } \\
{\left[\mathrm{J} / \mathrm{cm}^{2}\right]}\end{array}$ & $\begin{array}{c}\text { Opt. Pen- } \\
\text { etr. Depth } \\
\text { [nm] }\end{array}$ \\
\hline This work/ 100fs & $0.070 \pm 0.005$ & $15.8 \pm 1$ \\
This work/ 2ps & $0.074 \pm 0.005$ & $13.9 \pm 0.5$ \\
Mannion [22]/ 150fs & $0.065 \pm 0.005$ & $20.2 \pm 0.06$ \\
Nedialkov [20]/ 100fs & 0.06 & 22 \\
Harzic [12]/ 100fs & 0.021 & 17 \\
Harzic [12]/ 2ps & 0.03 & 10
\end{tabular}


optical penetration depth for both investigated pulse durations are shown and also compared to data from literature in Table 2.

It should be mentioned that the threshold fluences presented here are calculated based on the average fluence. Therefore, the actual local threshold fluence should be about $0.14 \mathrm{~J} / \mathrm{cm}^{2}$. The value is identical for both pulse durations in this work and also very close to the results from Mannion [22] and Nedialkov [20]. Harzic [12] obtained a much lower value but extrapolated to gain those values. With respect to the optical penetration depth, all values are in the same range between $10-22 \mathrm{~nm}$. Both Harzic and this work show that an increasing pulse duration decreases the optical penetration depth.

A comparison of the graphs for 2 ps and 100 fs for very high fluences of more than $50 \mathrm{~J} / \mathrm{cm}^{2}$ show that the ablation depth is higher in the 2 ps case. The use of the second laser system (Amphos) mostly yields the same results as expected. The only difference is visible in the beginning of the steep rise of the ablation depth at the transition from region II to III. As mentioned before, this might be caused by the different spatial pulse shape. Consequently, a different fraction of the area is exposed to the melt supported ablation. In region III this course is coinciding again. Both graphs created with 2 ps pulses do not feature the "plateau" which is visible for the pulse duration of $100 \mathrm{fs}$ in region III in Figure 1. In this case the intensity is one order of magnitude lower, thus less scattering of the laser pulses occur.

The investigation of the ablation depth obtained with the use of a process gas is more focused on the higher fluence regime and is carried out for three different spot sizes. In region I and II similar or only slightly lower ablation depths are measured while in region III the course of the graph diverges with respect to the other graphs. For the highest tested fluences, only about $50 \%$ of the ablation depth in comparison to a no gas process is reached. The process gas seems to push a bigger fraction of the ablated material back onto the surface which only affects the melt supported ablation regime. This effect seems to be stronger for the ablation depth, because the ablated volume is less than $25 \%$ lower in that case (see Figure 5). A similar observation has been made by Dausinger [16] when comparing the ablation depth and ab-

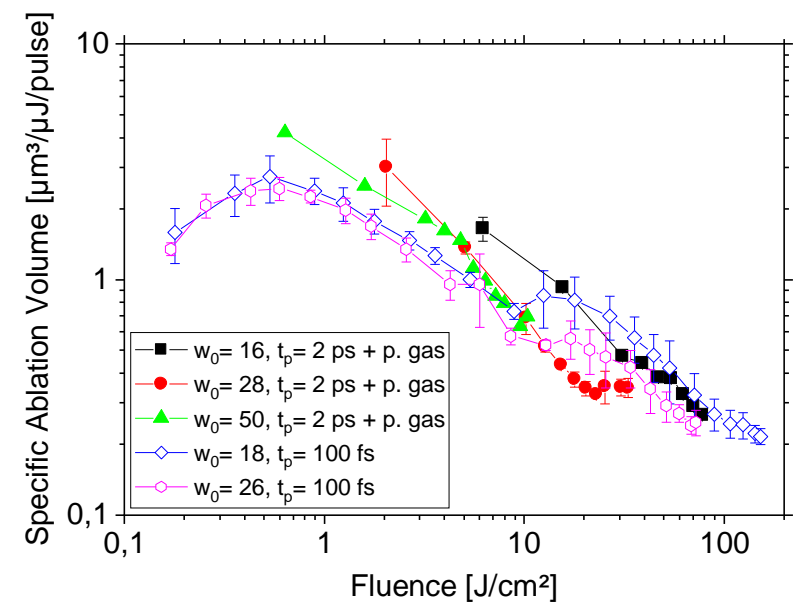

Fig. 5 Specific ablation volume per pulse as a function of the fluence for stainless steel (Libra: $\lambda=800 \mathrm{~nm}, \mathrm{t}_{\mathrm{p}}=100 \mathrm{fs}, \mathrm{f}=5 \mathrm{~Hz}$. Amphos: $\lambda=1030 \mathrm{~nm}, \mathrm{t}_{\mathrm{p}}=2 \mathrm{ps}, \mathrm{f}=1000 \mathrm{~Hz}$ ).

lation volume in ambient pressure and vacuum. For high fluences, the ablation volume is similar in both cases while the ablation depth is dependent on the surrounding pressure. Dausinger explained this behavior by a cold atmospheric plasma that is ignited at those high intensities. The radiation is mainly scattered away from the center of the Gaussian beam and decreasing the peak fluence in the center thereby. But the total energy deposited on the surface is only reallocated and therefore does not change the ablation volume. In Figure 5 the ablation volume per energy is shown to make the volumes obtained with different spot sizes more comparable to each other. The experiments with process gas and the experiments with $100 \mathrm{fs}$ pulse duration for reference are shown. The specific ablation volume can also be interpreted as a measure for the ablation efficiency. At about $0.6 \mathrm{~J} / \mathrm{cm}^{2}$ the well described (i.e. [30]) efficiency maximum can be observed followed by a decrease by one order of magnitude for an increasing fluence up to $100 \mathrm{~J} / \mathrm{cm}^{2}$. All displayed experiments have roughly the same course. The experiments with $100 \mathrm{fs}$ pulses display a constant efficiency in the range of $10 \mathrm{~J} / \mathrm{cm}^{2}$ which corresponds to the rise of the ablation depth at the transition to region III. This clearly shows that with reaching $10 \mathrm{~J} / \mathrm{cm}^{2}$ the ablation mechanism changes. As this
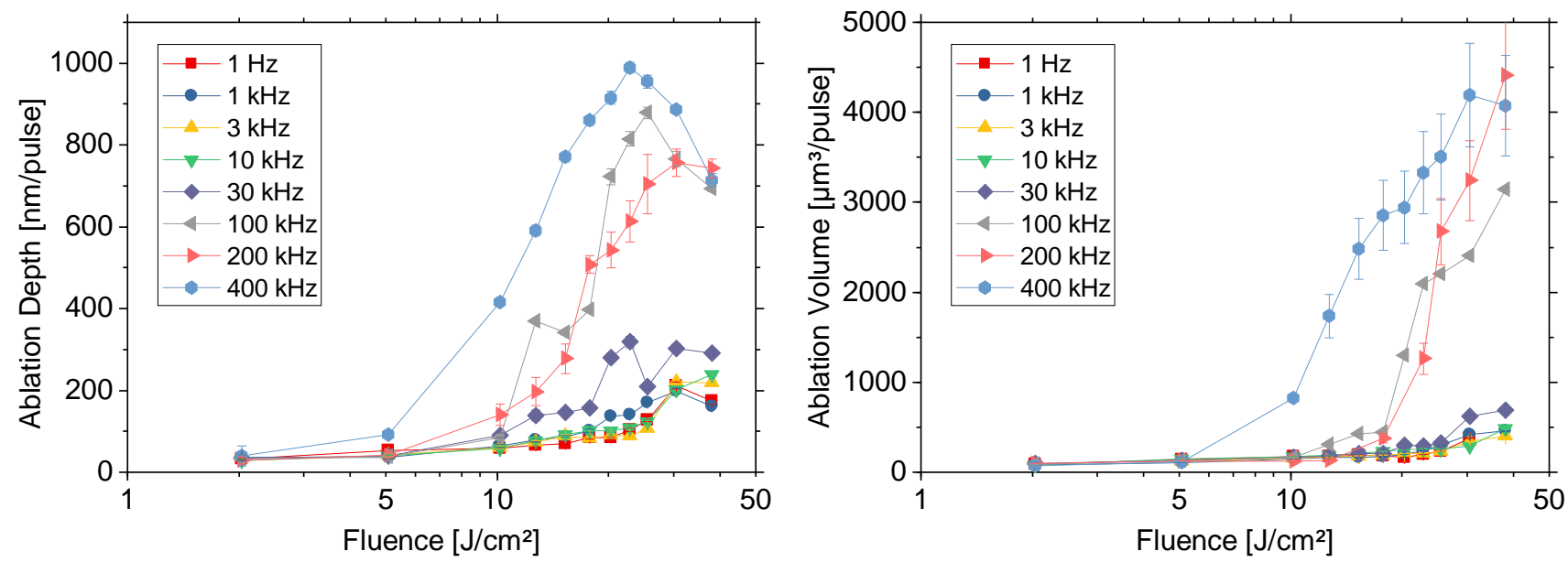

Fig. 6 Ablation depth and ablation volume per pulse as a function of the fluence for stainless steel for different repetition rates (Amphos: $\lambda$ $=1030 \mathrm{~nm}, \mathrm{t}_{\mathrm{p}}=2 \mathrm{ps}, \mathrm{w}_{0}=28 \mu \mathrm{m}, 100$ pulses $)$. 

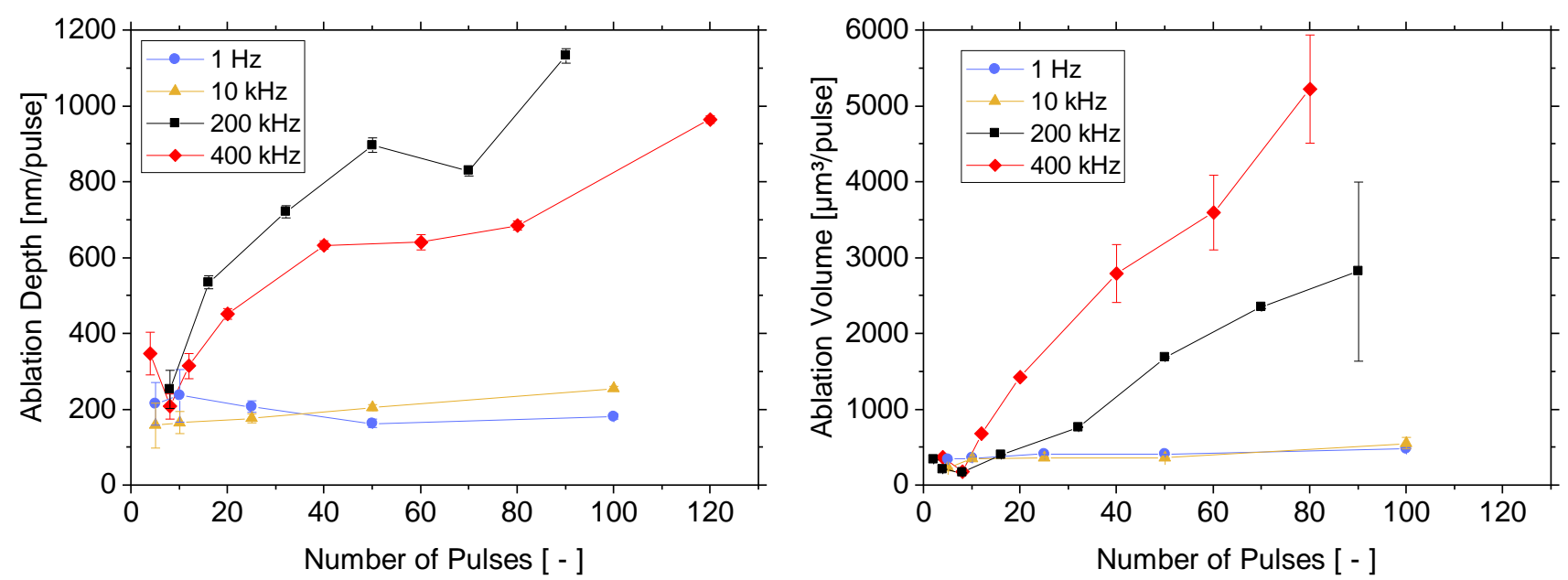

Fig. 7 Ablation depth and ablation volume per pulse as a function of the number of pulses for stainless steel for different repetition rates (Amphos: $\lambda=1030 \mathrm{~nm}, \mathrm{t}_{\mathrm{p}}=2 \mathrm{ps}, \mathrm{w}_{0}=28 \mu \mathrm{m}, \mathrm{F}=39 \mathrm{~J} / \mathrm{cm}^{2}$ ).

is the only visible change in the graph, this supports the conclusion that there is only one change of the ablation mechanism which is a melt supported ablation. Despite the significant lower ablation depth of the experiments that utilize process gas, the ablated volume is less influenced as seen in the graph.

\subsection{Heat accumulation at few pulses}

In the graph in Figure 6 the effect of an increased repetition rate on the ablation rate per pulse is shown. Both the ablation depth and ablation volume is displayed as a function of the fluence for repetition rates up to $400 \mathrm{kHz}$. Comparing both graphs leads again to the conclusion that the ablation volume is a more stable parameter to investigate the ablation rate when a melt supported ablation is present. For repetition rates up to about $10 \mathrm{kHz}$ no significant increase can be observed in the ablation rate, hence confirming that the results obtained before have not been altered by a repetition rate of $1 \mathrm{kHz}$. For $30 \mathrm{kHz}$, an increased ablation due to heat accumulation can be identified for high fluences over $10 \mathrm{~J} / \mathrm{cm}^{2}$ the first time. When further increasing to the maximum of $400 \mathrm{kHz}$, a huge increase of the ablation rate can be observed even though only 100 pulses are deposited. The ablation depth increases by a factor of $\sim 5$ while the ablation volume even exhibits a rise by a factor of $\sim 10$. In this case the heat accumulation is already present for fluences below $5 \mathrm{~J} / \mathrm{cm}^{2}$. In this set of experiments, the heat accumulation causes an increase of the ablation rate at an average power of about $50 \mathrm{~W}$ for $\mathrm{w}_{0}=28 \mu \mathrm{m}$.

Beside the repetition rate the amount of pulses also has an influence on the increase of the ablation rate per pulse (as the heat accumulation increases for more pulses). The effect of an increased number of pulses that are deposited on the sample is shown in Figure 7. For less than 15 pulses the ablation depth has high variations due to surface incubation effects that mainly change the surface structure. The ablation volume is also affected by this as a lower ablation volume is displayed in this region which supports the statement.

In addition, the statement at the beginning, that a minimum number of 25 pulses should be used to reach a steady and reproducible ablation rate, is confirmed again. For more than about 15 pulses, the ablation rate is roughly proportional for an increasing number of pulses.

\section{Conclusion}

In this study the ablation rate of stainless steel by means of a few ultrashort laser pulses up to $150 \mathrm{~J} / \mathrm{cm}^{2}$ is investigated in detail. The ablated areas are examined by the ablation depth and volume, and with WLI and SEM images. Also, the influence of the other adjustable parameters on the ablation rate has been investigated.

It has been shown in detail that a second logarithmic ablation regime, as indicated by literature, is not visible in the experiments. WLI images of the produced holes show an onset of a melt layer already at a mean fluence of $\sim 1.3 \mathrm{~J} / \mathrm{cm}^{2}$ which has not been described before. Hence, the potential electron diffusion ablation regime is superimposed from the beginning on by a melt supported ablation. Based on the graphs that illustrate the ablation rate and the WLI \& SEM images, three regions are defined:

- Region I is identical with the first ablation regime in which the optical penetration depth determines the ablation rate.

- In region II (starting at about $1 \mathrm{~J} / \mathrm{cm}^{2}$ ) both the vaporization dominated ablation for low fluences and the melt supported ablation are present due to the Gaussian intensity distribution of the beam. Reaching a fluence of about $10 \mathrm{~J} / \mathrm{cm}^{2}$, the ablation depth displays a steep rise which marks the beginning of the domination of the melt supported ablation and determines the transition to region III.

- In region III the ablation is fully melt supported, as the entire radiated area is covered by a melt layer. When investigating region III, the ablation volume is the suitable parameter to describe the ablation rate. Due to the existence of a liquid phase, melt dynamics lead to a high variation of the ablation depth while the volume still shows a monotone rising graph.

Both investigated pulse durations of $100 \mathrm{fs}$ and 2 ps behave in the same manner in terms of the ablation rate. Only slight differences between two different laser sources that might partly be caused due to the different wavelength and the spatial beam shape are observed 
The influence of other parameters on the ablation rate has also been investigated in detail in this paper and gives some remarkable results:

- The use of coaxial process gas has a strong influence on the ablation rate. The ablation depth decreases by about $50 \%$ while the ablation volume is decreased by about $25 \%$ compared to an ablation process without process gas. This means that the shape of the ablated depression also changes significantly when process gas is used.

- Different spot sizes lead to different ablation depths per pulse. This finding is contrary to the assumption of the two-temperature model that only the fluence determines the ablation depth. Also, it questions whether results obtained with considerably different spot sizes are comparable.

At higher fluences or higher repetition rates, an increase of the ablation rate due to heat accumulation occurs already at a few pulses and can lead to a significant rise. For fluences of about $40 \mathrm{~J} / \mathrm{cm}^{2}$, an increased repetition rate results in a higher ablation rate per pulse already for 15 pulses. With a rising number of pulses, both the ablation depth and volume increases roughly linearly. For 100 pulses with $40 \mathrm{~J} / \mathrm{cm}^{2}$ at $400 \mathrm{kHz}$, heat accumulation leads to an increase of the ablation rate by a factor of 5 to 10 . The start of heat accumulation is found to be based on the average power which is in these experiments roughly $50 \mathrm{~W}$ at 100 pulses and $\mathrm{w}_{0}=28 \mu \mathrm{m}$.

In conclusion, in ultrafast ablation of stainless steel for fluences greater than $1 \mathrm{~J} / \mathrm{cm}^{2}$, the generation of melt and the rise of the ablation rate due to heat accumulation should always be taken into consideration already at very few laser pulses. However, higher ablation rates or an increased depth of drilled holes can be achieved by means of high fluences. Combining this with a low fluence finishing process has the potential of still employing the advantages of ultrafast ablation at an elevated process speed.

\section{Acknowledgments}

Parts of this work have been funded by the German Federal Ministry for Economic Affairs and Energy (BMWi) within the grant for the project COORETEC (Grant No. 03ET7027A) and by the German Research Foundation DFG under Grant No. PO 591/41-1.

\section{References}

[1] W. Sibbett, A. Lagatsky, and C. Brown: Opt. Express, 20, (2012) 6989.

[2] W. Kautek and J. Krüger: Proc. SPIE, 2207, (1994) 600.

[3] B. C. Stuart, M. D. Feit, A. M. Rubenchik, B.W. Shore and M. D. Perry: Phys. Rev. Lett., 74 12, (1995) 2248.

[4] J. Ihlemann, A. Scholl, H. Schmidt and B. WolffRottke: Appl. Phys. A, 60, (1995) 411.

[5] P. P. Pronko, S. K. Dutta, J. Squier, J. V. Rudd, D. Du and G. Mourou: Opt. Comm., 114, (1995) 106.

[6] A. Luft, U. Franz, A. Emsermann and J. Kaspar: Appl. Phys. A, 63, (1996) 93.

[7] S. Preuss, A. Demchuk and M. Stuke: Appl. Phys. A, 61, (1995) 33.

[8] C. Momma, B. N. Chichkov, S. Nolte, F. von Alvensleben, A. Tünnermann, H. Welling and B. Wellegehausen: Opt. Comm., 129, (1996) 134.
[9] B. N. Chichkov, C. Momma, S. Nolte, F. von Alvensleben and A. Tünnermann: Appl. Phys. A, 63, (1996) 109.

[10] S. Nolte, C. Momma, H. Jacobs, A. Tünnermann, B. Chichkov, B. Wellegehausen and H. Welling: J. Opt. Soc. Am. B, 14, (1997) 2716.

[11] S. Nolte, C. Momma, A. Tünnermann, B. Chichkov, B. Wellegehausen and H. Welling: Laser and Optoelectronic, 3 29, (1997) 82.

[12] R. Le Harzic, D Breitling, M. Weikert, S. Sommer, C. Föhl, S. Valette, C. Donnet, E. Audouard and F. Dausinger: Appl. Surf. Sci., 249, (2005) 322.

[13] B. Neuenschwander, F. F. Bucher, C. Nussbaum, B. Joss, M. Muralt, U. W. Hunziker and P. Schuetz: Proc. SPIE, 7584, (2010) 75840R.

[14] B. Jaeggi, B. Neuenschwander, M Schmid, M. Muralt, J. Zuercher and U. Hunziker: Phys. Proc., 12, (2011) 164.

[15]B. Neuenschwander, B. Jaeggi, M. Schmid, V. Rouffiange and P.E. Martin: Proc. SPIE, 8243, (2012) 824307.

[16]F. Dausinger: RIKEN Review No. 50: Focused on Laser Precision Microfabrication, (2002) p.77. (LPM 2002)

[17] I. Minagareev: "Ultrafast Dynamics of Melting and Ablation at Large Laser Intensities", (Göttingen, 2009) p.74

[18] J. Lopez, G. Mincuzzi, R. Devillard, Y. Zaouter, C. Hönninger, E. Mottay and R. Kling: J. Laser Appl., 27, (2015) S28008.

[19] J. Schille, L. Schneider and U. Loeschner: Appl. Phys. A, 120, (2015) 847.

[20] N. N. Nedialkov, S. E. Imamova and P. A. Atanasov: J. Phys. D: Appl. Phys., 37, (2004) 638.

[21] B. Jaeggi, B. Neuenschwander, M. Schmid, M. Muralt, J. Zuercher and U. Hunziker: Phys. Proc., 12, (2011) 164.

[22] P. T. Mannion, J. Magee, E. Coyne, G. M. O'Connor and T. J. Glynn: Appl. Surf. Sci., 233, (2004) 275.

[23] J. B. Nielsen, J. M. Savolainen, M. S. Christensen and P. Balling: Appl. Phys. A, 101, (2010) 97.

[24] J. P. Negel, A. Loescher, D. Bauer, D. Sutter, A. Killi, M. A. Ahmed and T. Graf: Opt. Express, 23, (2015) 21064.

[25] P. Russbueldt, T. Mans, J. Weitenberg, D. Hoffmann, R. Poprawe: Opt. Lett., 35, (2010) 4169.

[26]D. J. Förster, R. Weber and T. Graf: Proc. 18th Int. Symp. on Laser Precision Microfabrication, Toyama, (2017) A120.

[27] D. J. Förster, R. Weber, D. Holder and T. Graf: Opt. Express, 26, (2018) 11546.

[28] C. Woerz, Christoph, G. Reichardt, M. Liewald, E. Zahedi, R. Weber, T. Graf: Dry Met. Forming OAJ FMT 4, (2018).

[29] B. Lauer, B. Jaeggi and B. Neuenschwander: Phys. Proc., 56, (2014) 1047.

[30] L.V. Zhigilei, Z.Lin, D.S. Ivanov: J. Phys. Chem. C, 113, (2009) 11892.

(Received: June 18, 2019, Accepted: November 25, 2019) 